\title{
Product Reference Number
}

National Cancer Institute

\section{Source}

National Cancer Institute. Product Reference Number. NCI Thesaurus. Code C99287.

The identifier assigned to a firm's product. 